Cahiers « Mondes anciens »

ANCIENS

Histoire et anthropologie des mondes anciens

$4 \mid 2013$

Journées doctorales ANHIMA 2010 et 2011

\title{
Représenter la victoire
}

Les hydries, objets de récompense dans la céramique attique du $v^{e}$ s. av. n. è.

\section{Gwenola Cogan}

\section{OpenEdition}

Journals

Édition électronique

URL : http://journals.openedition.org/mondesanciens/1020

DOI : $10.4000 /$ mondesanciens. 1020

ISSN : 2107-0199

Éditeur

UMR 8210 Anthropologie et Histoire des Mondes Antiques

Référence électronique

Gwenola Cogan, « Représenter la victoire», Cahiers « Mondes anciens » [En ligne], 4 | 2013, mis en ligne le 01 juillet 2013, consulté le 10 décembre 2020. URL : http://journals.openedition.org/ mondesanciens/1020; DOI : https://doi.org/10.4000/mondesanciens.1020

Ce document a été généré automatiquement le 10 décembre 2020.

\section{(c) (i) (9)}

Les Cahiers "Mondes Anciens » sont mis à disposition selon les termes de la licence Creative Commons Attribution - Pas d'Utilisation Commerciale - Pas de Modification 4.0 International. 


\section{Représenter la victoire}

Les hydries, objets de récompense dans la céramique attique du $v^{\mathrm{e}}$ s. av.n. è.

\section{Gwenola Cogan}

1 En Grèce ancienne les pratiques athlétique et hippique constituent une part importante de l'activité des citoyens, le grand nombre de représentations où les jeunes gens s'adonnent à la palestre et à son univers en témoigne. Ces pratiques constituent une part de la formation des éphèbes à la citoyenneté. Dans ces conditions, il n'est pas surprenant que ce sujet ait donné lieu à une vaste iconographie vasculaire, notamment en Attique au $\mathrm{V}^{\mathrm{e}}$ siècle av. n. è. ${ }^{1} \mathrm{Il}$ n'est pas question ici d'étudier le corpus des scènes qui représentent les compétitions athlétiques ou hippiques, ni même le cadre de la palestre et du gymnase, mais d'examiner plus précisément le mode de représentation de la victoire de l'athlète dans un concours. Cette victoire est représentée et mise en scène notamment par la présence des objets de récompense, ceux remis à l'issue des concours. Leur étude permet de mieux appréhender leur fonction symbolique et leur valeur performative. Ces récompenses sont en effet nombreuses et variées, couronnes, trépieds, bandelettes, coupes ou encore chaudrons ${ }^{2}$. Toutefois, dans le cadre de cet article je souhaiterai m'intéresser plus particulièrement au cas de l'hydrie ${ }^{3}$. Néanmoins, pour comprendre l'importance de cet objet comme prix de la victoire, il est nécessaire de s'y intéresser non seulement dans le cadre athlétique, gymnique ou hippique, mais il convient également de regarder, au-delà du thème de l'agôn, l'ensemble des domaines d'utilisation des hydries pour cerner leur valeur et leur sens symbolique ${ }^{4}$. Ce faisant, il devient alors possible de déterminer les fonctions de l'hydrie dans des contextes singuliers d'usage, et ainsi de révéler une valeur, un rôle, précis et récurrent qu'elle conserve quelles que soient les situations dans lesquelles elle est représentée sur les vases. Cette approche se distingue ainsi du travail d'Eurydice Képhalidou qui a précédemment mené une étude sur la représentation de la victoire ${ }^{5}$. Dans son ouvrage, les différents types de prix font chacun l'objet d'un chapitre. L'hydrie y tient bien sûr une place particulière. Elle y est traitée et considérée intrinsèquement comme un objet de valeur tant symbolique que marchande, un objet de prestige et de prix dans tous les sens du terme. Mais l'hydrie y est étudiée 
dans un seul contexte, celui de l'agôn, et ce au milieu d'une série de représentations de la victoire. Le choix se porte ainsi sur un contexte d'emploi des objets, celui de la victoire, et non pas sur un objet et ses multiples emplois ${ }^{6}$. Ma démarche est donc inverse, considérant l'objet lui-même et examinant les différents contextes iconographiques dans lesquels il est représenté. Cette étude se place ainsi dans la suite d'une série de travaux menés ces dernières années par François Lissarrague et ses étudiants qui examinent et analysent la place, le rôle et le sens de l'objet dans le champ de l'image ${ }^{7}$. Les représentations de l'hydrie sur les vases semblent ainsi renvoyer à des domaines très éloignés les uns des autres. Néanmoins, au vu de ces différents contextes, l'hydrie conserve au contraire un sens précis dans chacun d'entre eux. Que ce soit dans le cadre de la victoire athlétique, ou bien dans l'importante série des femmes à la fontaine, ou encore sur les représentations du rapt de Polyxène par Achille, ou, en dernier lieu, lorsqu'elle sert d'objet de rançon comme c'est le cas pour le corps d'Hector par exemple, l'hydrie conserve une signification commune. Dans chacun de ces cas, la transition et le passage d'un état à un autre sont l'élément commun que l'objet permet de matérialiser, l'hydrie en devient alors le signe visible, tangible.

\section{Les hydries comme athla, prix de la victoire}

2 Le contexte agonistique est aisément reconnaissable grâce notamment à la présence de Nikè auprès des athlètes ou des récompenses qui lui sont destinées. Néanmoins, les gestes de Nikè à l'égard des concurrents, mais aussi la façon dont elle tient les objets, ainsi que son attitude peuvent changer sur ces représentations selon les cas. De fait, il existe toute une série de nuances dans l'action de Nikè et de sa relation autour des objets de la victoire. Tout d'abord, Nikè est seule avec les objets de récompense ; tandis qu'ensuite les athlètes, ou bien les épreuves elles-mêmes, sont représentés sur les vases. Nous étudierons dans un premier temps, le cas où Nikè est seule avec les objets de la victoire qu'elle manipule encore parfois sur le vase; avant de nous attarder, dans un second temps, sur la représentation de l'épreuve elle-même, ainsi que sur la question de la forme du vase et du jeu d'écho qui peut alors s'installer entre le décor et le vase.

\section{Nikè en vol}


Fig. 1 : Hydrie attique à figures rouges

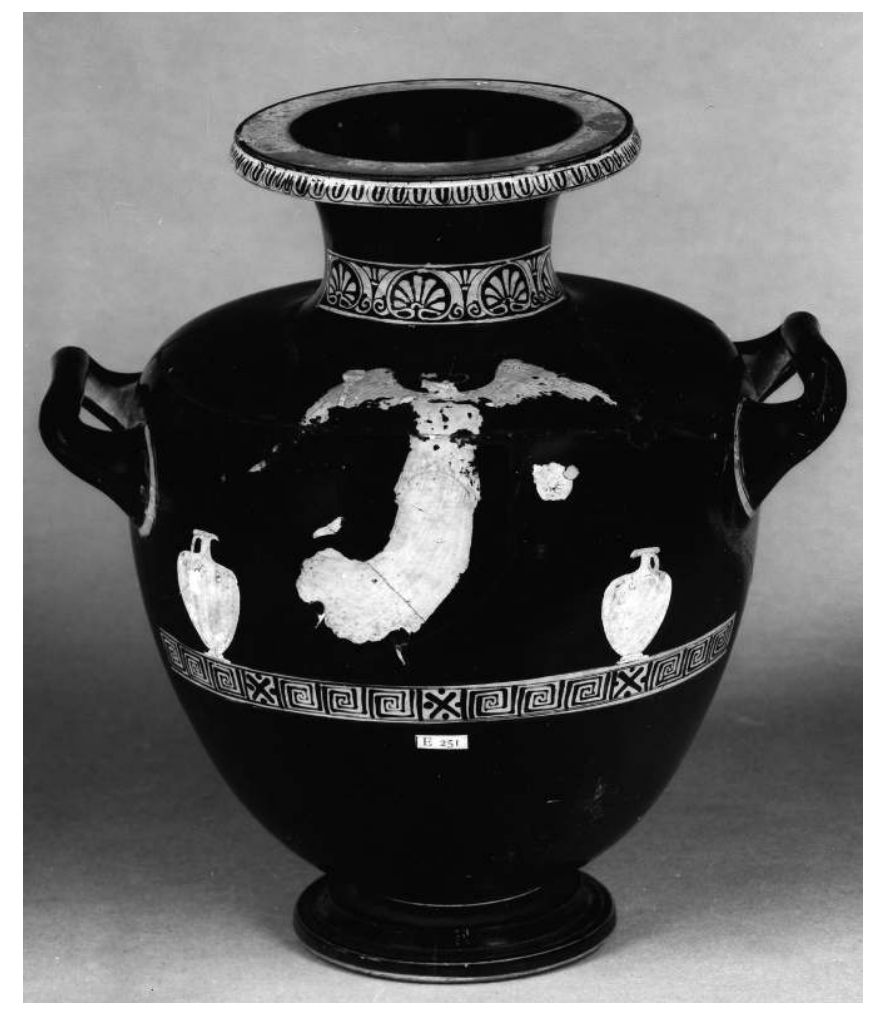

Londres, British Museum E251 (1772,0320.163)

(c) The Trustees of the British Museum

3 Sur plusieurs hydries la déesse vole parmi des objets de récompense, elle les porte et les transporte. Sur une hydrie du British Museum, datée des années 500-450, la déesse tient un vase à la main, elle vole entre deux hydries posées par terre et qui encadrent la scène (Fig. 1) ${ }^{8}$. Une inscription «kalos/kale: Nikè» également figure sur le vase ${ }^{9}$. Cette inscription renvoie à la " belle victoire ", expression à double sens ici, qu'il s'agisse de la déesse qui apporte le prix et/ou de l'exploit accompli par l'athlète lors de l'épreuve qui le vit triompher. L'athlète a lui aussi rencontré la belle Victoire. 
Fig. 2 : Amphore du peintre de Berlin

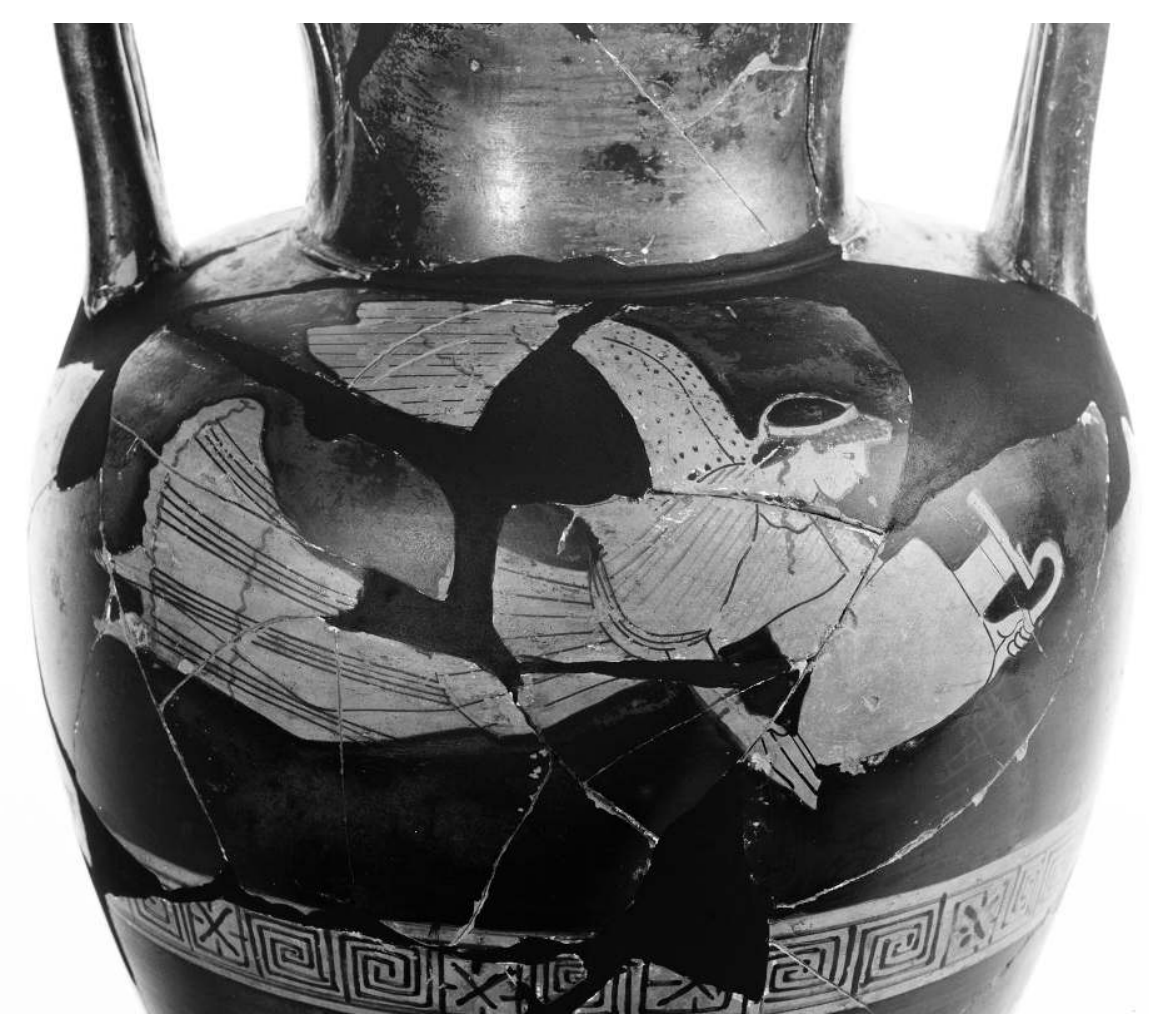

Oxford Ashmolean Mus. 1930.36

(C) Ashmolean Museum University of Oxford

De la même manière, une amphore du peintre de Berlin montre, sur la face $A$, Nikè en plein vol portant une hydrie vers l'athlète représenté sur la face B (Fig. 2) ${ }^{10}$. Une hydrie du peintre d'Oreithyia met également en scène une Nikè en vol et qui porte, là encore, une hydrie. Dans ce cas, la face $B$ du vase ne donne pas de représentation complémentaire de l'athlète ${ }^{11}$. Néanmoins, entre le vase lui-même, l'hydrie, et la scène figurée, il convient de remarquer la mise en abyme qui renvoie ainsi le spectateur au contexte agonistique, tout autant qu'à la victoire de l'athlète. Il y a un jeu d'écho entre le vase et son motif décoratif, entre la forme de l'objet et la signification de sa représentation. Un dernier exemple enfin, à partir des fragments d'un cratère en calice de Larissa dont la décoration est double et organisée sur deux registres horizontaux. Ce cratère possède la particularité de représenter quatre épreuves, deux musicales sur le registre supérieur, deux épreuves gymniques sur le registre inférieur ${ }^{12}$. Pour ce qui concerne le registre supérieur, on identifie, sur la face A, d'une part un aulode qui reçoit une couronne, et d'autre part Nikè, en vol, qui lui apporte une hydrie, probablement de bronze ${ }^{13}$. Sur la face B du même registre, face qui est très lacunaire, on parvient toutefois à distinguer une autre Nikè tout à droite, derrière le pilier d'une estrade sur laquelle un duo de musicien se tient face à un homme assis, barbu et couronné. Il est ici impossible d'aller plus avant dans l'identification de l'épreuve et des prix, bien qu'il y soit aussi question de la remise des prix aux musiciens victorieux. Le registre inférieur présente quant à lui deux épreuves gymniques. En face A, une scène de combat, lutte ou pancrace ${ }^{14}$; tandis que sur la face $B$, une hoplitodromie se court au rythme de l'aulos. Cette dernière scène est elle aussi très lacunaire. En revanche, la face A, dans sa partie inférieure, nous permet d'identifier les personnages : Nikè, dont on distingue le bout des ailes, ainsi que la tête tournée vers la 
droite, soit à l'opposé du vainqueur. Le vainqueur Lysiklès, dont on ne voit que le sommet du crâne et les bandelettes qui lui ceignent la tête, le juge, Antiphanès, tête levée, proclame le nom du vainqueur; alors que Thrasyklès, le perdant, s'éloigne, le regard tourné vers le sol. La lacune, qui englobe Nikè et Lysiklès, rend impossible toute indentification du prix que la déesse apporte au vainqueur. Ce cratère de Larissa offre une belle présentation des différents types de concours, musicaux et athlétiques, qui sont ici associés et mis en parallèle dans une sorte de complémentarité de l'un et de l'autre. Néanmoins, l'état fragmentaire de cette hydrie nous met face à bien des interrogations tout en proposant peu de pistes de réponse. Quelle était l'épreuve de musique pour laquelle Nikè apporte une belle hydrie ? Dans quelle épreuve gymnique les deux athlètes s'affrontèrent-ils? Et qu'allait recevoir Lysiklès des mains de "sa » Nikè? Une hydrie identique à celle que sa consœur apporte au musicien chanceux? Autant de questions auxquelles il est difficile de répondre de manière définitive. Le détail des scènes et la profusion des noms inscrits sur le vase en font explicitement une commande pour commémorer un événement particulier, une victoire importante pour le commanditaire ${ }^{15}$

5 Les cas ici exposés attestent la présence et l'action bienfaitrice de Nikè, mais cette action n'est encore que suggérée. La divinité est associée au vainqueur, elle se dirige vers lui les mains pleines des objets de la reconnaissance. Mais il existe un répertoire des représentations de Nikè qui va au-delà, par exemple lorsqu'elle dépose dans les mains de l'athlète les récompenses tant attendues.

\section{Nikè donatrice}

6 Comme dans une sorte de crescendo dans les représentations de la victoire athlétique et de Nikè, l'iconographie offre également un type de mise en scène où Nikè et les athlètes se font face. La divinité et les vainqueurs se trouvent alors comme sur un pied d'égalité, au moment où ces derniers reçoivent les prix qu'elle a transportés pour eux. La remise en main propre des objets de prix constitue en outre une source d'inspiration pour les peintres. Ainsi, sur un fond de coupe où l'athlète tient dans sa main gauche son strigile, son paquetage et son alabastre, il reçoit des mains de la déesse les prix qui récompensent et matérialisent sa victoire ${ }^{16}$. Nikè tient, elle aussi dans la main gauche, plusieurs objets qu'elle remet au vainqueur : un lébès et une phiale qu'elle tient par l'anse. Sur la scène figure encore un troisième objet remis en signe de victoire. Il s'agit d'une hydrie qui est ainsi déposée entre les deux personnages. Nikè l'a-t-elle précédemment remise aux pieds du vainqueur? L'impression générale qui se dégage de l'image, c'est que le vaserécompense, probablement en bronze, appartient déjà à l'athlète. En effet, il convient d'observer combien l'hydrie est à proximité de l'athlète, véritablement à ses pieds, comme s'il en avait d'ores et déjà la possession exclusive et la jouissance personnelle. Sur ce vase, le nom de la divinité est inscrit, en une sorte d'insistance d'autant plus forte, jouant sur le double sens du mot nikè ${ }^{17}$; à la fois celui de la divinité et celui de la victoire de l'athlète. En outre, ce dernier est couronné, marque supplémentaire de son nouveau statut, enfin sa proximité avec Nikè qui lui remet en main propre ses prix, sans compter que l'un d'entre eux est déjà déposé à ses pieds. Il y a là une multiplication des signes du statut du vainqueur, l'expression de sa valeur, de son mérite, ainsi que de l'importance de son exploit, de son athlos. Si l'on s'attache aux objets de la récompense, on constate qu'ils font partie des plus prestigieux, ne laissant, dès lors, aucun doute sur le caractère 
exceptionnel de la victoire et la nature des exploits accomplis. Il y a de la part de l'artiste une volonté de jouer sur la valeur tant de l'athlos, l'exploit, que sur celle des athla, des prix dont il ne cesse de mettre en avant le caractère prestigieux.

\section{La représentation de l'épreuve}

7 Certains vases, quant à eux, mettent en scène les athlètes concourant pour le prix qui est exposé et mis en valeur dans le champ du vase. La composition offre donc à voir l'athlos au moment où il est en train de s'accomplir, mais elle offre aussi une vision de l'athlon, le prix, la récompense, qui attend que le vainqueur soit désigné par les dieux, dont la présence est révélée par certains signes. Il en est ainsi d'un cratère en cloche du groupe de Polygnotos ${ }^{18}$. Sur la face A, deux jeunes gens disputent une course aux flambeaux ${ }^{19}$, ils arrivent l'un derrière l'autre à l'autel au pied duquel est déposée une hydrie. Derrière cet autel, un homme se tient debout, drapé dans un vêtement à manche courte ${ }^{20}$. Il attend les concurrents, il les regarde courir, d'un signe de la main, il désigne d'une part l'hydrie présente à sa droite, prix de la victoire ; tandis que d'autre part, avec la main gauche il indique le pied d'olivier qui se dresse de l'autre côté, derrière l'autel. Cette représentation d'une branche d'olivier symbolise la présence divine parmi les hommes, qui par ailleurs honorent la déesse de leur effort par la course qu'ils se livrent ${ }^{21}$. Sur la face B sont représentés trois hommes drapés dans leur manteau. L'homme de gauche tient un strigile qu'il tend au personnage central qui pourtant lui tourne le dos, puisqu'il fait lui-même face au personnage de droite qui, quant à lui, tient un bâton, somme toute une scène ordinaire de la vie du citoyen grec ${ }^{22}$. D'un côté, la scène de la face A met en évidence l'épreuve de la course aux flambeaux. Celle-ci se dispute le plus souvent dans des compétitions civiques qui mettent en concurrence des équipes formées sur la base des tribus de la cité, et dans lesquelles s'affrontent les jeunes éphèbes, avant qu'ils n'entrent officiellement dans la catégories des andres, les citoyens de plein de droit ${ }^{23}$. Les courses aux flambeaux et la mise en concurrence des tribus de la cité ont un rôle important dans les rites, car c'est avec la torche du vainqueur que l'on allume le feu qui servira pour le sacrifice et pour le rituel de la fête ${ }^{24}$. Dans ces conditions, le prestige de la victoire et de l'objet qui la matérialise a en premier lieu une valeur religieuse pour l'ensemble de la tribu qui est ainsi honorée ${ }^{25}$. Ici l'association entre le prix au pied de l'autel d'une part, et le juge, ou l'archonte-roi, ainsi que la branche d'olivier d'autre part, montre, s'il en était besoin, que cette valeur religieuse est celle qui prime parmi les différents sens qui sont attachés aux objets de prix. Par ailleurs, les jeunes gens font ici la preuve de leur mérite personnel qui leur permet de justifier et de légitimer leur passage du groupe des éphèbes à celui des citoyens, et pour ce faire la course et les cérémonies religieuses sont nécessaires. L'hydrie est également une façon de marquer cette transition au moment même où elle a lieu.

Dans la même perspective, un skyphos du peintre de Thésée fait figurer les prix au moment où se dispute la compétition ${ }^{26}$. Sur le vase, le peintre semble vouloir associer la pratique hippique et les devoirs du citoyen, à moins que ce ne soit une allusion à une autre épreuve athlétique. En effet, ce vase nous montre sur la face A une course de char qui voit s'affronter deux quadriges luttant pour remporter les prix exposés sur trois hautes colonnes qui, de cette manière, dessinent une sorte de centre de piste. Trois grands dinoi couronnent donc ces colonnes et doivent être compris comme représentant les prix de la victoire. Sur la face B, trois hoplites en rang portent leur bouclier et leur 
lance ; ils sont encadrés par de grandes palmettes de part et d'autre de la scène. Toutefois, ces hoplites ne semblent pas être prêts à l'action, les deux personnages de gauche se retournent vers le dernier personnage à droite, alors même qu'ils semblent devoir avancer vers la gauche. Leur attitude ressemble fort à celle de l'indécision ou à de l'attente. Quelle action vont-ils entreprendre? S'agit-il des préparatifs pour une hoplitodromie, ou bien est-ce la préparation à la guerre ? Dans les deux cas, les vêtements qui tombent de leur bras ne sont propices ni à une course en armes, ni à l'action sur un champ de bataille ${ }^{27}$. Il est aussi possible que nous soyons dans le cadre d'un défilé ou d'une revue, ou encore d'un concours de beauté lors d'un agôn, et pour lequel la panoplie doit être la plus impeccable possible pour montrer la valeur des citoyens de la polis ${ }^{28}$. Tous trois portent des casques corinthiens dont un seul cimier diffère, celui de l'hoplite de droite. Faut-il considérer cette différence comme un indice en faveur d'un concours de beauté ? Un autre indice inciterait à penser qu'il s'agit également d'un concours, qu'il soit de beauté ou qu'il s'agisse d'une hoplitodromie: c'est la présence de deux hydries représentées sous chacune des anses du skyphos. L'une est représentée de côté ; tandis que l'autre est peinte de dos. Si les meneurs de char se disputent les dinoi, il est fort probable que les hoplites qui regardent chacun vers les anses, concourent quant à eux pour les hydries présentes entre les deux scènes. Ce skyphos renvoie ainsi aux activités du citoyen, la participation à des épreuves hippiques d'une part, et d'autre part une épreuve directement liée au statut civique, à la capacité de s'armer et de représenter la cité par la possession d'une panoplie. Le statut d'hoplite est ici mis en valeur, les activités qui lui sont liées sont honorées par l'octroi d'un prix, ici une ou deux hydries. Ces deux exemples montrent de quelle manière les activités athlétiques sont réservées à ceux qui possèdent le statut de citoyen ${ }^{29}$. Par conséquent, les objets remis comme prix sont investis de la même valeur de définition du statut civique. L'objet ne vient pas valider un état, mais il permet d'attester cet état. Il est ainsi la preuve de l'appartenance au groupe civique.

9 Si les récompenses sont représentées sur les vases, soit avec Nikè, soit associées au moment de l'épreuve et à l'athlos qui est en train de s'accomplir, il existe aussi des cas où les vases eux-mêmes sont des prix remis aux vainqueurs des épreuves.

\section{Le vase comme prix}

Lorsque ce n'est plus seulement Nikè qui est présente auprès du prix, ce peut être la divinité honorée par le concours, comme c'est le cas pour les amphores panathénaïques. La présence de la déesse sur l'amphore, alors que le vase lui-même constitue le prix de la victoire, laisse entrevoir la valeur symbolique très forte qui est donnée à la récompense. Pourtant, parmi les plus anciennes amphores panathénaïques, qui datent de la seconde moitié du $\mathrm{VI}^{\mathrm{e}}$ siècle, d'autres types de prix, en plus de l'amphore, figurent sur le vase. De fait, que peut bien signifier la représentation d'un autre prix sur un vase qui n'est autre que le contenant de la récompense la plus importante, l'huile sacrée des oliviers d'Athéna? On trouve ainsi une amphore du groupe de Bulas, désormais à New York au Metropolitan Museum, sur laquelle figure en face A la déesse Athéna, et en face B, un athlète assis sur une hydrie ${ }^{30}$. S'il est certain que l'amphore elle-même, parce qu'elle contient l'huile issue des oliviers sacrés d'Athéna, constituait un prix remis au vainqueur de l'une des épreuves des Panathénées, il ne faut pas non plus être surpris de voir d'autres objets de prestige lui être associés comme récompense. Dans une inscription, 
certes postérieure de plus d'un siècle à cette amphore, on apprend que pour toutes les épreuves des Panathénées qui se couraient en équipe, c'est-à-dire entre les tribus athéniennes, d'autres prix que les amphores étaient remis tels que des bœufs, le plus souvent, ou des hydries ${ }^{31}$. Sur le vase du groupe de Bulas, on distingue que le vainqueur, assis sur son hydrie, a en outre la tête ceinte d'une bandelette blanche qui témoigne de sa victoire et des honneurs que la foule lui a rendus. Il tient une torche à la main, ce qui fait de lui un coureur de lampadédromie. Or, l'inscription, mentionnée plus tôt, précise que ce sont des hydries que l'on remettait à la tribu victorieuse dans l'une de ces courses aux flambeaux $^{32}$. Sans que l'on puisse dire qu'il y ait eu une continuité entre notre amphore panathénaïque de la seconde moitié $\mathrm{du} \mathrm{vI}^{\mathrm{e}} \mathrm{s}$. et cette inscription de la première moitié du $\mathrm{IV}^{\mathrm{e}} \mathrm{s}$., il reste intéressant de porter notre attention sur cette correspondance entre la représentation sur l'amphore et le contenu de l'inscription au sujet des objets décernés à l'issue des épreuves.

11 Dans chacun des différents cas étudiés ici, nous avons pu observer que des éléments très symboliques et très significatifs donnaient un sens précis aux objets de prix, qu'ils rendaient explicites le contexte agonistique. Qu'il s'agisse de la présence de Nikè à côté des objets, ou bien qu'elle les apporte et les dépose aux pieds ou dans les mains des vainqueurs, la reconnaissance officielle se fait au moment de la remise du prix. Toutefois, ces objets peuvent aussi être associés à l'action même de l'athlos, lorsque les jeunes gens courent vers l'autel ou bien que les chars usent l'hippodrome, les objets prennent alors tout leur sens d'athlon. Dans chacun de ces cas, les hydries représentées, souvent sur des vases qui sont eux-mêmes des hydries, renvoient à un moment de transition et de passage d'un état à un autre, de la reconnaissance de cette transition. Que ce soit le passage de simple concurrent à celui de vainqueur, ou bien plus particulièrement celui des jeunes éphèbes qui s'apprêtent à accéder pleinement au statut de citoyen. Ces scènes figurent en effet des cas où la jeunesse est idéalisée. L'absence de barbe, signe de la maturité des hommes, fait ici écho aux vers de Pindare concernant le glabre des jeunes ageneioi pour lesquels la participation aux concours est un élément de la formation des futurs citoyens ${ }^{33}$ . Dans le cadre agonistique l'hydrie ne doit donc plus seulement être considérée comme une récompense à la valeur prestigieuse, car elle marque, tout comme dans le domaine féminin, le changement d'état de celui qui la reçoit et en aura désormais l'usage.

Ce rôle de l'hydrie est en effet associé depuis bien longtemps au passage et à la transition d'un état à un autre en contexte féminin. C'est principalement dans le cadre de la jeune fille à la fontaine que cette fonction est bien attestée, au moment où la nymphè accède elle aussi à un nouveau statut.

\section{Une histoire d'eau : I'hydrie et « les femmes à la fontaine »}


Fig. 3 : Hydrie du peintre AD

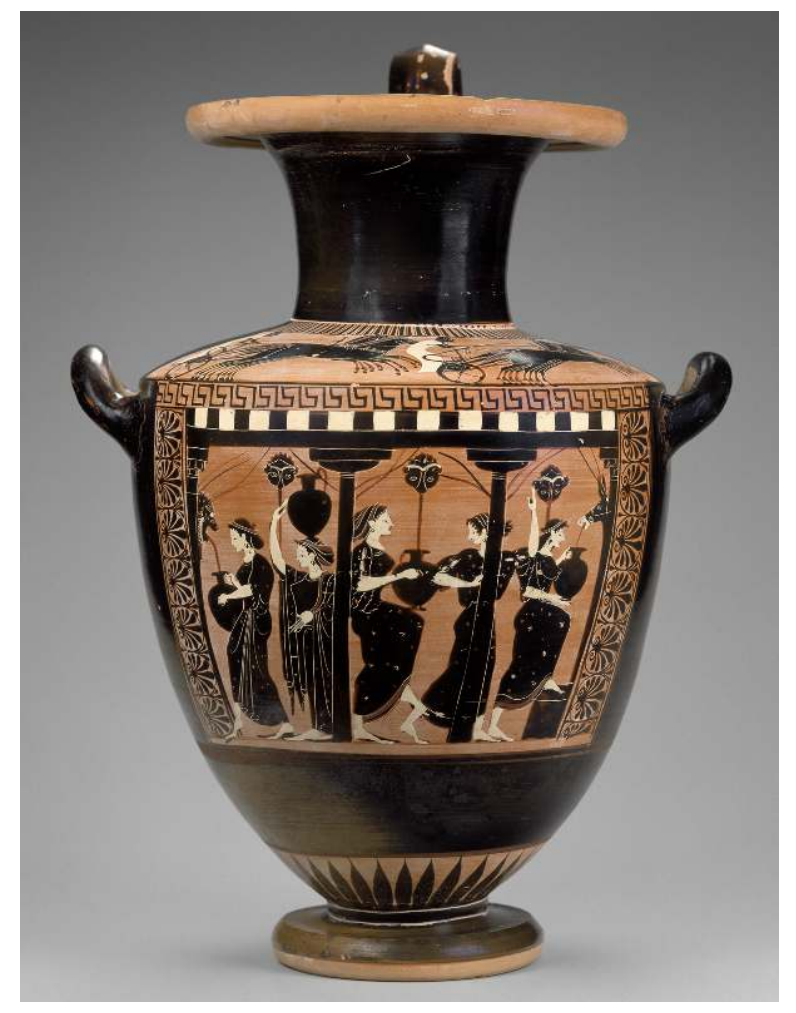

Boston, Museum of Fine Arts 61.195 (525-475, ca 510)

(c) Museum of Fine Arts, Boston

L'iconographie des femmes à la fontaine a inspiré de nombreux chercheurs qui ont eu à cœur d'explorer ce vaste champ de représentation ${ }^{34}$. La plupart de ces travaux renvoient à une analyse typologique des scènes en faisant apparaître leur structure, ainsi qu'en proposant une interprétation précise de chacun des éléments figurés. Apparaît alors une typologie singulière et très codifiée des femmes à la fontaine qui, de fait, suivent un parcours scrupuleux. Si on cherche à identifier et définir ces femmes, on se rend compte qu'il s'agit bien plus de parthenoi ou de nymphai que de gynai ${ }^{35}$. Ainsi en est-il d'une hydrie $\mathrm{du}$ peintre $\mathrm{AD}$ désormais à Boston sur laquelle on peut voir les jeunes filles danser et chanter autour des fontaines tout en s'adonnant avec joie à leur tâche (Fig. 3$)^{36}$. Les femmes à la fontaine sont figurées sur la panse de l'hydrie; tandis que sur l'épaule sont représentés des hommes et des activités liées au monde masculin ${ }^{37}$. Les exemples sont nombreux d'hydries, car ce sont exclusivement des hydries, sur lesquelles de telles scènes sont représentées et où la correspondance se fait, où l'écho résonne, entre le masculin et le féminin, entre l'épaule et la panse du vase ${ }^{38}$. 
Fig. 4 : Hydrie attique à figures noires du groupe de Léagros

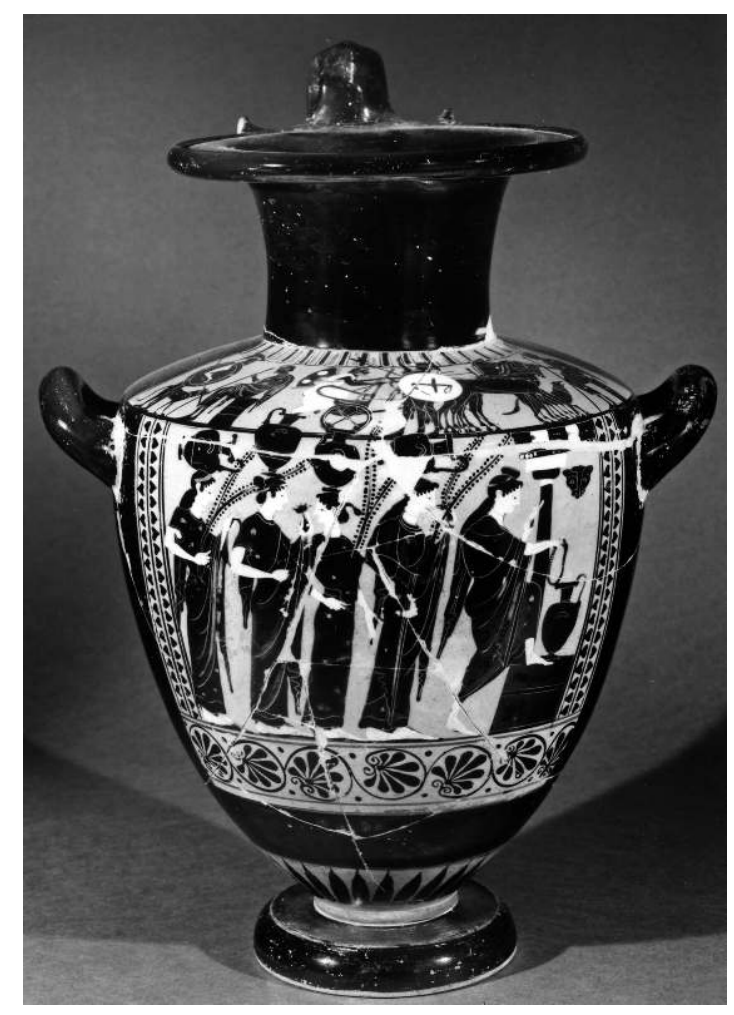

Londres, British Museum B337 (1867,0508.957)

(c) The Trustees of the British Museum

Chacun de ces vases présente des éléments propres qui les distinguent les uns des autres, mais ils possèdent néanmoins de nombreux points communs, leur iconographie renvoie à un univers singulier qui dit quelque chose de précis sur les rapports entre les jeunes filles et les hommes qu'elles rencontrent, sur les relations des unes aux autres. Considérons tout d'abord les représentations des jeunes filles, qui figurent un idéal de la jeunesse et de la beauté39. Ce sont des parthénoi, des nymphai, richement parées de vêtements ouvragés, portant de nombreux bijoux, boucles d'oreille, collier, bracelets ${ }^{40}$. Elles portent également des bandeaux dans les cheveux, tiennent des fleurs à la main (Fig. 4) ${ }^{41}$. Ces scènes nous transportent dans un contexte idéalisé où les jeunes filles partagent des moments agréables dans un environnement chatoyant où la nature et les animaux sauvages sont présents, témoignant ainsi de la présence d'Artémis dans leurs activités ${ }^{42}$. 
Fig. 5 : Hydrie à figures noires du groupe de Léagros

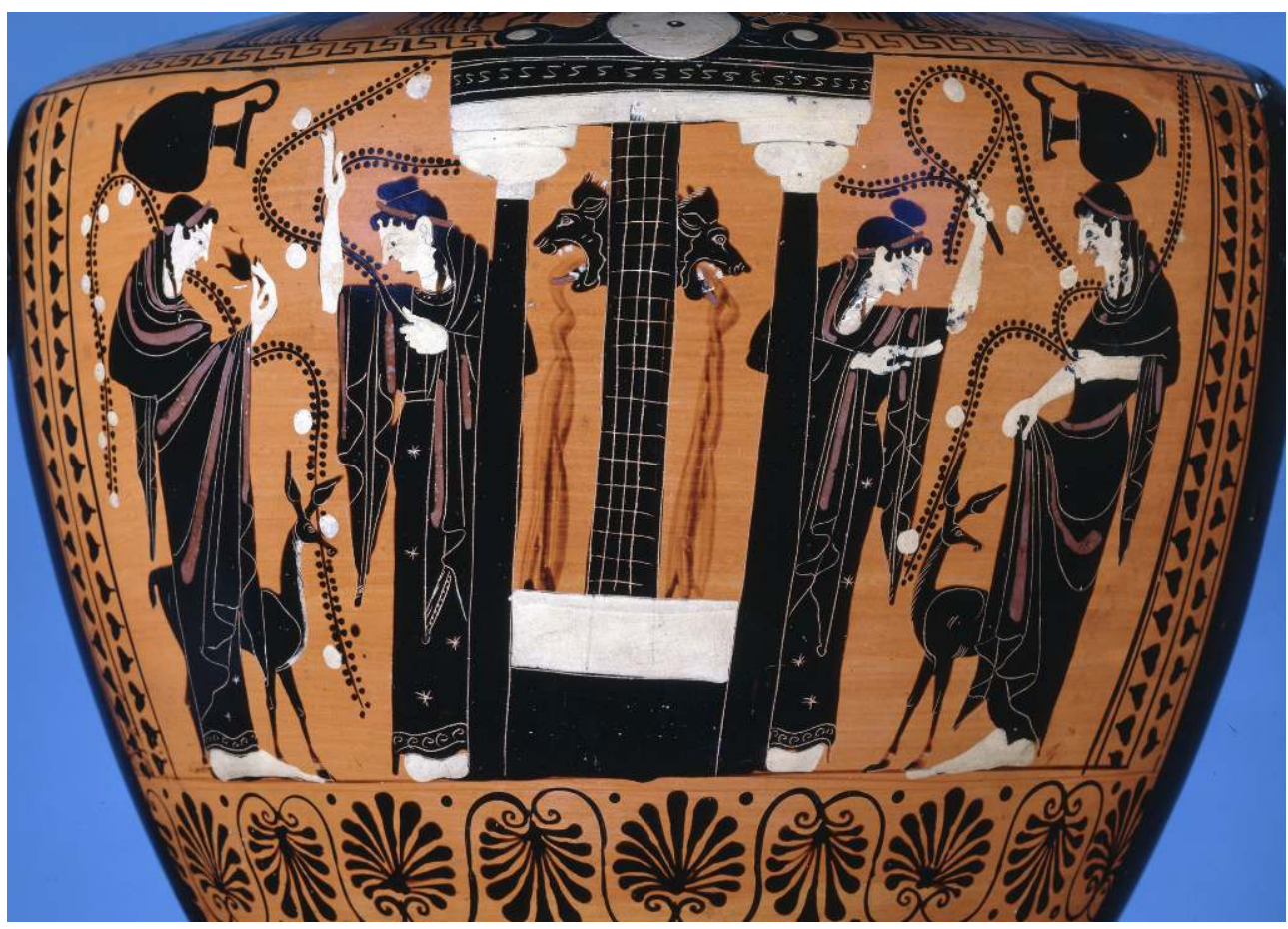

Londres, British Museum B335 (1843,1103.56)

(c) The Trustees of the British Museum

15 Cette présence animale est parfois discrète, n'apparaissant que dans l'architecture par le biais des bouches de fontaine qui sont en forme de lion, ou de lionne ${ }^{43}$, ainsi qu'en forme de tête d'âne ou de sangliers ${ }^{44}$, mais la nature sauvage se fait parfois plus explicite avec la présence de biches, qui symbolisent la virginité et l'innocence, l'animal d'Artémis, celui de la chasse (Fig. 5) ${ }^{45}$. Nous sommes dans un univers féminin, mais qui est encore celui d'un monde indompté, non codifié, par le mariage et la condition d'épouse. Le passage n'a pas encore eu lieu, alors que certains signes l'annoncent déjà. L'âne est ainsi l'animal qui tire le char de la jeune épousée vers sa nouvelle demeure ${ }^{46}$. C'est donc la jeune femme qui s'apprête à changer d'état, de statut et l'hydrie en est le signe ${ }^{47}$. Cet univers féminin renvoie alors explicitement aux différents types d'activités masculines représentés sur l'épaule. Si les peintres séparent ces activités sur deux parties distinctes du vase, ils ne les excluent pas les unes des autres, mais expriment au contraire une sorte de complémentarité des domaines masculin/féminin. Les hommes sont donc présents en tant qu'athlètes qui prouvent leur valeur physique, leur supériorité, leur excellence ${ }^{48}$, en tant que guerriers qui se préparent à lutter pour sauvegarder la cité, leur foyer ${ }^{49}$. Ce type d'hydrie correspond à une volonté de lier les deux sphères masculine/féminine dans un même objectif commun, celui de franchir les étapes de la vie tout en maintenant la stabilité et la fertilité du foyer comme celles de la cité. La fontaine, l'eau fertile qui remplit l'hydrie et qui ainsi peut assurer la pérennité du foyer, renvoient explicitement au thème du mariage, celui de l'union consenti ou parfois même arrachés ${ }^{50}$.

Cette dernière catégorie, celle de la démonstration de force masculine face à la jeune femme à la fontaine, est aussi présente dans l'iconographie des hydries ${ }^{51}$. Observons ainsi l'épaule d'une hydrie du British Museum sur laquelle figure la poursuite d'Achille après Troïlos, alors que Polyxène a laissé choir son hydrie, qui s'est brisée, et qu'elle-même 
s'enfuit face à la violence dont fait preuve le héros ${ }^{52}$. Dans certaines de ces représentations, l'hydrie de Polyxène est brisée, signe de l'arrêt de son activité, de la rupture des étapes traditionnelles de la vie des jeunes filles, du basculement de son destin ${ }^{53}$. Toutefois, la figuration de cet épisode pourtant violent de l'épopée troyenne ${ }^{54}$, donne parfois lieu à des scènes calmes et paisibles, où le désir du héros apparaît comme chaste et bienveillant, comme sur une hydrie de l'Ermitage où Achille est accroupi derrière la fontaine et observe patiemment Polyxène (Fig. 6) ${ }^{55}$.

Fig. 6 : Hydrie à figures rouges du peintre de Berlin

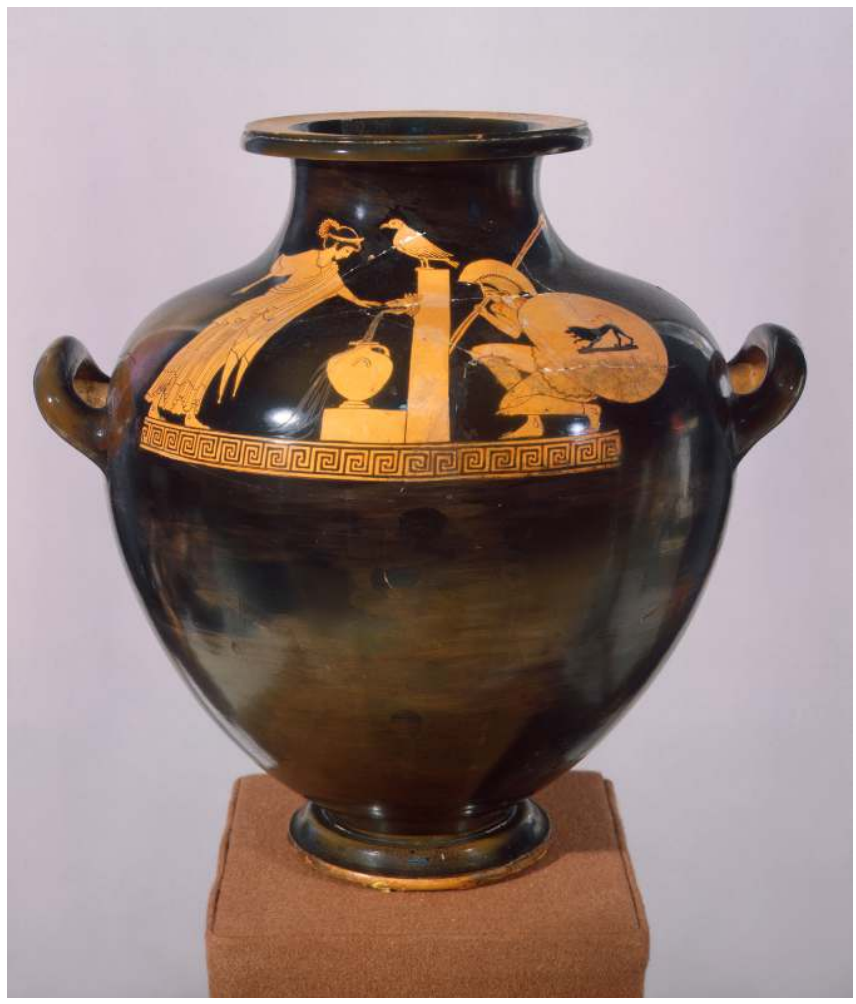

Saint-Pétersbourg, Musée de l'Ermitage 628 (ST 1588-200)

(c) The State Hermitage Museum / Vladimir Tereberin, Leonard Kheifets, Yuri Molodkovets

Bien que les deux scènes représentent le même épisode, il faut bien reconnaître que l'atmosphère qui s'en dégage diffère totalement l'une de l'autre. L'hydrie de l'Ermitage offre sans doute la représentation la plus métaphorique de l'union de la femme et de l'homme, lorsque l'eau de la fontaine remplit et fait déborder l'hydrie lui donnant sa plénitude, car si l'eau est bien la source de fertilité, c'est pourtant grâce à l'hydrie que le foyer peut être abreuver et se maintenir. En outre, la représentation d'Achille et de Polyxène est une ode à la jeunesse et à la beauté. Ils représentent tous deux l'idéal du héros et de la jeune fille. L'hydrie apparaît donc comme l'objet qui symbolise cette union, il s'agit d'un objet de prestige et de valeur, un objet qui marque une transition, celle qui correspond au passage à l'âge adulte, aux responsabilités.

Cette transition et ce changement sont représentés de manière métaphorique et idéalisée aussi bien dans le contexte agonistique que dans celui des femmes à la fontaine. Cependant, il reste un type de représentation dans lequel l'hydrie est utilisée à la fois comme objet de prestige à l'importante valeur marchande, et comme signe d'un changement d'état, symbolisant alors l'ultime transition. 


\section{L'hydrie objet de valeur, objet d'échange ?}

19

Nous retiendrons ici l'exemple d'un skyphos de Brygos, conservé à Vienne ${ }^{56}$. Sur ce vase, Priam et sa suite viennent réclamer auprès d'Achille le corps $d^{\prime} H_{e}{ }^{2}{ }^{3}{ }^{57}$. Pour qu'Achille accepte de rendre le corps du défunt, Priam apporte avec lui des objets précieux issus de ses propres coffres. Parmi ces objets figurent des hydries, évoquées tant dans le texte d'Homère que sur le vase qui nous occupe. Si les échanges se font traditionnellement avec des objets de prestige et de grande valeur marchande, ceux qu'offre Priam à Achille sont les mêmes que ceux que Nikè remet au vainqueur sur le fond de coupe perdu du Musée d'Athènes ${ }^{58}$. Les objets du trésor de Priam sont, comme pour les vainqueurs, ceux de la reconnaissance d'un statut social, ceux du prestige, du renom et de la valeur personnelle, celle d'Achille, celle d'Hector, celle de Priam, celle d'un vainqueur aux agônes. Le héros comme l'athlète est idéalisé. Achille vainqueur d'Hector, est l'athlète par excellence, celui qui accomplit les exploits, mais c'est aussi celui qui provoque la mort de son rival. Celui-ci franchit alors le dernier passage, celui pour lequel l'hydrie est également utilisée afin de préparer le corps à la sépulture, afin de réparer la souillure de la mort, mais aussi celle de l'outrage subi. Elle est nécessaire à la purification du corps pour permettre que le dernier passage se fasse, celui dans la mort, afin que le défunt trouve sa place et le repos dans l'Hadès.

20 De fait, l'hydrie est ici recouverte de plusieurs sens qui tous se font écho. Objet de prestige utilisé dans les transactions marchandes, l'hydrie permet la reconnaissance de la valeur et du mérite de celui qui la possède. Cette reconnaissance permet ainsi d'accéder au statut le plus envié, celui d'épouse, celui de citoyen, celui de l'athlète victorieux, celui du héros épique. Enfin, si l'hydrie accompagne son possesseur dans les étapes de sa vie, elle est aussi nécessaire à son dernier voyage afin de l'accompagner dans sa dernière demeure. En offrant, entre autres, des hydries à Achille, Priam lui rappelle l'ensemble de ces significations, la valeur dont le héros doit faire preuve pour ne pas priver Hector de son statut.

\section{Conclusion}

21 À l'issue de cette étude sur la représentation des hydries et l'analyse de leur présence dans des contextes si divers que celui de la victoire athlétique, celui des jeunes femmes à la fontaine ou bien encore celui de l'échange de biens dans une tractation marchande, il convient d'établir le bilan des différents rôles de l'hydrie dans ces contextes. De fait, l'hydrie est riche d'une polysémie qui lui permet de fonctionner non pas seulement comme marqueur d'un statut particulier, mais également comme révélateur des changements de statut de l'individu qui entre en possession d'une hydrie. Dans le contexte agonistique, l'hydrie, en plus d'être un objet de prestige, met en évidence le passage d'un statut à un autre, de simple concurrent à celui de vainqueur, c'est-à-dire de favori des dieux. Dans le cas des jeunes filles à la fontaine, elle est aussi l'expression de la fin de l'innocence nubile pour entrer dans l'état d'épouse et de mère où la responsabilité du foyer et de sa pérennité va prendre toute son ampleur, alors même que les hommes se présentent comme des héros triomphant sur les pistes des hippodromes, ou comme des guerriers prêts à risquer leur vie pour défendre l'intégrité de leur foyer et plus largement celle de leur cité. Enfin, dans le cadre de l'épopée, l'hydrie garde son statut d'objet de 
prestige et de grande valeur marchande, mais dans le cadre de la rançon d'Hector, elle symbolise aussi la volonté de Priam de rendre à son fils les derniers hommages, de lui permettre de changer là encore de statut et de condition, de gagner un nouvel état, celui de la plus grande félicité, mais seulement à condition que le corps souillé soit enfin purifié, et pour cela ce sont les hydries qui sont utilisées pour accomplir les rites funéraires dans lesquels elles sont employées pour apporter l'eau lustrale. L'hydrie est donc un objet qui, en plus de ses attributions matérielles, marque le passage, la transition d'un état à un autre. On observe toutefois, que ces changements d'état suivent une gradation vers toujours plus de reconnaissance et d'accomplissement de soi. L'athlète est alors vainqueur, l'éphèbe accède la citoyenneté ${ }^{59}$, la jeune femme devient une épouse, Hector trouve le repos aux Enfers. Ce sont là différentes étapes qui permettent aux Hommes de s'accomplir et de s'acquitter de leur devoir envers les dieux, envers l'ordre divin et le kosmos. Les hydries en sont notamment l'un des symboles.

\section{BIBLIOGRAPHIE}

BEAZLEY J. D. (1956), Attic Black Figures Vases, Oxford.

- (1963), Attic Red Figures Vases ${ }^{2}$, Oxford.

- (1982), Addenda, Oxford.

- (1989), Addenda², Oxford.

- (1971), Paralipomena, Oxford.

BOEGEHold A. L. (1996), « Group and Single Competitions at the Panathenaia », dans Neils J. éd., Worshipping Athena, Panathenaia and Parthenon, Madison, p. 95-105.

CARTER J. B. et MORRIS S. P. (1996), The Ages of Homer : A Tribute to Emily Townsend Vermeule, Austin.

DUCREY P. (1985), Guerre et guerriers dans la Grèce antique, Paris.

ESPosito A. M. et DE tommaso G. éd (1993), Vasi Attici, Museo Archeologico Nazionale di Firenze, Florence.

DIETRICH N. (2010), Figur ohne Raum? Bäume und Felsen in der attischen Vasenmalerei des 6. und 5. Jh. v. Chr., Berlin.

GHERCHANOC F. (2008), « Nudités athlétiques et identités en Grèce ancienne », dans Gherchanoc F. et Huet V. éd., S'habiller, se déshabiller dans les mondes anciens, Mètis N.S.6, Paris, p. 75-101.

GIGLIOLI G. Q. (1951), « Lampadedromia », Archologia Classica 3, Rome, p. 147-162.

GÜNTNER G. et al. (1997), Mythen und Menschen, Griechischen Vasenkunst aus einer deutschen Privatsammlung, Mayence.

JOUANNA J. (1994), « La roue tourne et le sportif court... avec une couronne sur la tête », BCH 118, p. 35-49.

JÜNTHER J. (1924), « Lampadédromia », RE XII, 1, col. 57-577. 
KEPHALIDOU E. (1996), Nikitis, Eikonographiki meleti tou archaiou ellinikou athlitismou, Thessalonique.

KNITTLMAYER Br. (1997), Die Attische Demokratie und ihre Helden, Dartstellungendes trojanischenSagenkreise im 6. Jh. v. Chr., Heidelberg.

KYLE D. G. (1992), «The Panathenaic Games : Sacred and Civic Athletics », dans Neils J. éd., Goddess and Polis, The Panathenaic Festival in Ancient Athens, Madison.

LATACZ J. et al. (2008), Homer, Der Mythos von Troia in Dichtung und Kunst, Munich-Bâle.

LISSARRAGUE F. (1985), « Paroles d'images : remarques sur le fonctionnement de l'écriture dans l'imagerie attique », dans Christin A. M. éd., Écritures II, Paris, p. 71-93.

- (1992), « Graphein : écrire et dessiner », dans Bron Chr. et Kassapoglou E. éd., L'image en jeu de l'Antiquité à Paul Klee, Lausanne, p. 189-203.

- (2001), Greek Vases, The Athenians and their Images, New York.

- (2006), « De l'image au signe : objets en représentation das l'imagerie attique », Cahiers du CRH, avril, $\mathrm{n}^{\circ} 37, \mathrm{p} .11-24$.

- (2007), « Ajax, corps et armes », dans Colpo I, Favaretto I., Ghedini F éd., Iconografia 2006, Gli Eroi di Omero, Rome, p. 21-32.

- (2008), « Corps et armes : figures grecques du guerrier », dans Dasen V et Wilgaux J. éd., Langages et métamorphoses du corps dans le monde antique, Rennes, p. 15-27.

MANAKIDOU E. (1992/3), « Athenerinnen in Schwarzfiguren Brunnenheusszenen », Hephaistos 11/12, p. 51-91.

MANFRINI ARAGNO I. (1992), « Femmes à la fontaine : réalité et imaginaire », dans, Bron Chr.et Kassapoglou E. éd., L'image en jeu de l'Antiquité à Paul Klee, Lausanne, p. 127-148.

MILLER M. C. (1989), « The Ependytes in Classical Athens » Hesperia 58, p. 313-329.

NEILS J. (1992), Goddess and Polis, The Panathenaic Festival in Ancient Athens, Madison.

PEDLEY J. G. (1992), Greek Art and Archeology, New York.

SABETAI V. (2009), « The Poetics of Maidenhood : Visual Constructs of Womanhood in Vase-

painting ", dans Zanker P., CVA Bayerische Akademie der Wissenschaften, Band IV, p. 103-114.

SIMON E. (1983), Festival of Attica : An Archeological Commentary, Winsconsin.

Stamatopoulou M. (2007), « Thessalian Aristocracy and Society in the Age of the Epinician ", dans Morgan C. et Hornblower S. éd., Pindar's Poetry, Patron, and Festival, from Archaic Greece to the Roman Empire, Oxford, p. 309-341.

TIVERIOS M. A. 1989, Perikleia Panathenaia, Athènes, (en grec, traduction allemande, Perikleische Panathenäen. Eine Krater des Malers von München 2335, Gutenberg, 2008).

VALAVANIS P. (1990), « La proclamation des vainqueurs aux Panathnées. À propos d'amphores panathénaïques de Praisos », BCH 114, p. 325-359, fig. 18.

\section{NOTES}

1. Il s'agira principalement dans le cadre de cette étude de céramique attique. Concernant la chronologie, sauf mention contraire, toutes les dates sont avant notre ère. 
2. Il n'était pas possible dans le cadre de l'intervention, ni dans celui de cet article, de traiter de la mise en scène et de la représentation de l'ensemble des objets de prix. Néanmoins, à la suite de cette première étude sur l'hydrie, d'autres études devraient suivre concernant la représentation iconographique de chacun des objets traditionnellement remis comme athlon afin de parvenir par la suite à une véritable analyse iconographique de l'ensemble des prix de concours dans la céramique attique classique.

3. Je remercie les organisateurs de cette journée doctorale "Autour de l'image: problèmes et méthodes» (10 avril 2010, Paris, INHA) de m'avoir ainsi donné l'opportunité de développer quelques pistes de réflexion sur ce sujet.

4. Une étude plus vaste de l'iconographie des objets de prix de concours mériterait d'être menée pour mettre en lumière les différents principes qui régissent à la représentation de la victoire et de ses attributs.

5. KePHALIDOU 1996.

6. Dans la même perspective, traitant d'un unique contexte et en examinant l'hydrie dans une thématique singulière, on peut également citer les travaux d'Ivonne MANFRINI ARAGNO 1992, p. 127-148 ; ceux d'Elini MANAKIDOU 1992/3, p. 51-92 ; de Victoria SABETAI 2009, p. 103-114.

7. Pour François Lissarrague on consultera les articles suivants qui mettent en avant la valeur symbolique et la sémiotique des objets dans la peinture sur vase : LISSARRAGUE 2006, p. 11-24 ; 2007 , p. $21-32 ; 2008$, p. 15-27. Concernant les thèses qu'il a dirigées ou dirige encore on citera par exemple: DiETRICH 2010. N. KeI, «L'esthétique des fleurs: kosmos, poikilia et charis dans la céramique attique du $\mathrm{VI}^{\mathrm{e}}$ et $\mathrm{du} \mathrm{V}^{\mathrm{e}}$ siècle av. J.-C.", thèse soutenue en 2010 ; V. ZACHARI, "Autour de l'autel: espace, temps et gestualité sur la céramique attique de la période archaïque et classique ", thèse en cours à l'EHESS.

8. Cf. CVA London, British Museum 6, pl. (376) 101.1 ; LIMC, VI, pl. 570, Nikè 113.

9. Sur l'interprétation, la fonction et le sens des inscriptions sur les vases, voir notamment François LISSARRAGUE 1985, p. 71-93; et sur la présence du terme «kalos » p. 73 et p. 87-88; et aussi LISSARRAGUE 1992, p. 189-203.

10. Cf. $A R V^{2}, 202.89$; KÉPHALIDOU 1996, p. 204 et pl. 40 Г76.

11. Hydrie du peintre d'Oreithyia, Varsovie MN 142288. Cf. CVA, Goluchow, Musee Czartoryski, 19, pl. (020) 20.2A.2B ; cf. ARV 2 , 496.8 ; et aussi Add., 122.

12. Cratère en calice du peintre de Munich 2335, Larissa Musée archéologique 86.101. Cf. TIVERIOS 2008, pl. 1-22. Voir aussi VALAVANIS 1990, p. 344, fig. 18 ; voir aussi NEILS 1992, p. 61, fig. 39 a et b ; KÉPHALIDOU 1996, p. 211 et pl. 47-48 Г97.

13. Pour le détail de cette face voir TIVERIos 2008, pl. 1-5.

14. Le vaincu que l'on peut voir s'éloignant tête basse ne porte pas de gant, il ne doit donc pas s'agir d'une épreuve de boxe.

15. Voir notamment TiVERIOS 2008, p. 83-89, où l'auteur fait une étude des noms qui sont inscrits sur le vase; voir également NEILS 1992, p. 61 qui reprend majoritairement les conclusions de T iverios. Voir aussi StAMATOPOULOU 2007, p. 335-336.

16. Coupe d'un successeur de Douris, datée des années 475-425, Athènes aujourd'hui perdu, cf. ARV ${ }^{2}, 1670.58$ bis ; KéPHALIDOU 1996, p. 207 et pl. 45 Г85.

17. Voir note 8 .

18. Cratère en cloche, attribué au groupe de Polygnotos par Beazley, ou bien à la manière du peintre de Peleus, Arthur M. Sackler Mus., Université d'Harvard, 1960.344. Cf. CVA, Baltimore, Robinson Collection 2, 34-35, pl. 290, 291, 47.2, 48.1 a-b. Cf. ARV ${ }^{2}, 1041.10$; Add., 156 ; Para., 443 ; KÉPHALIDOU 1996, p. 218, pl. $10 \Lambda 1$.

19. Sur la discussion autour de l'identification des diadèmes que portent les jeunes coureurs, voir notamment JOUANNA 1994, p. 35-49, et plus particulièrement p. 46-48 pour la représentation de la couronne pendant la course, fig. 5. L'hypothèse qu'il propose de reconnaître dans ces couronnes 
un élément de distinction des équipes entre elles me paraît tout à fait convaincante. Par ailleurs, sur un cratère athénien où Nikè vient remettre le bandeau de la victoire au coureur qui a triomphé sur le front duquel se trouve un diadème portant l'inscription suivante: ANTIOC. Les deux autres coureurs ont aussi des diadèmes portant des inscriptions. Cratère en cloche athénien anciennement collection Tyskiewicz, désormais au British Museum, 1898.7-16.6. Cf. ARV ${ }^{2}$, 1333.1 ; Para., 480 ; et voir aussi GLILIOLI 1951, p. 153, pl. XXXVIII ; KEPHALIDOU 1996, pl. 56. Г4 (A).

20. Sur ce type de vêtement appelé épendytes et utilisé notamment pour certaines cérémonies religieuses voir MILLER 1989, p. 313-329; pl. 54a, plus particulièrement pour ce vase voir p. 321 où l'auteur propose de reconnaître en l'homme debout derrière l'autel la figure de l'archonte roi, dont on sait qu'il présidait des courses aux flambeaux, cf. Aristote, Constitution des Athéniens, 57, 1. 21. Voir la description de ce vase par GLILIOLI 1951, p.152, pl. XXXVI, 2. La présence d'une branche d'olivier renverrait ainsi aux courses de flambeaux qui se courent durant les Panathénées, voir aussi l'association de la déesse avec Héphaïstos GLILIOLI 1951 p. 157-158.

22. Sur la question de l'association entre nudité athlétique et statut de citoyen voir notamment GHERCHANOC 2008, p. 85-86.

23. Voir notamment JÜNTHER 1924, col. 570-577 ; et aussi GIGLIOLI 1951, p. 147-162.

24. Voir SIMON 1983, p. 53 pour les différentes célébrations dans lesquelles ont lieu des lampadédromies à Athènes, p. 64 pour celles qui se déroulent lors des Panathénées et dont le feu pris sur l'autel sacré servira à allumer le feu du sacrifice. Voir aussi KYLE 1992, p. 96.

25. IG II ${ }^{2}, 2311,11.88-89$.

26. Skyphos du peintre de Thésée (505-495), collection privée. Voir GÜNTNER 1997, p. 53-55, nº 14.

27. Un autre élément qui laisse penser qu'il n'y a pas d'action guerrière en vue tient notamment dans l'impression de calme qui émane de la scène par opposition à la face $\mathrm{A}$ et la tension de l'épreuve qui se dispute. Il y aurait donc un dialogue entre les deux scènes qui se jouent sur le vase. Voir GÜNTNER 1997, p. 52.

28. Cf. IG $\mathrm{II}^{2}, 2311$, col. B, 1. 87, pour des épreuves lors des Panathénées ; et aussi IG $\mathrm{II}^{2}$ 956, 1. 48 ; IG II $^{2} 957$, 1l. 26-35 pour des épreuves d'euandria lors des Théseia. Pour des épreuves d'euandria à Elis cf. ATHÉNÉE, XIII, 565. Voir aussi BOEGEHOLD 1996, p. 97-98.

29. Sur ce point voir GHERCHANOC 2008, p. 87, où il est question de l'interdiction faite aux esclaves de pratiquer une activité gymnique, athlétique, car cette pratique est réservée aux citoyens.

30. Amphore Panathénaïque du groupe de Bulas, New York, Metropolitan Museum 41.162.52. Cf. CVA New York, Metropolitan Museum of Art 3, 40, pl. (580) 48.3-4; et aussi CVA Cambridge (MA) Fogg Musuem and Gallatin Collections, 88, pl. (388) 40.2A-B. Cf. ABV, 661.4 ; Para., 316.

31. IG II ${ }^{2} 2311$, col. B, 11. 83-93.

32. IG II 2311 col. B 1.89.

33. Pindare, Néméennes $5,6$.

34. J'emprunte ici le titre de l'article de MANFRINI ARAGNO 1992 et qui donne par ailleurs une bibliographie de départ sur la question. Voir aussi MANAKIDOU 1992/3, p. 51-91; SABETAI 2009, p. 103-114.

35. Sur ce point voir SABETAI 2009, p. 104.

36. Cf. CVA, Boston, Museum of Fines Arts 2, 23-24, pl. (915), 81. 1-3 ; cf. Add. ${ }^{2}, 91$; Para., 147.5 bis. MANAKIDOU 1992/3, p. 57, fig. 5. Sur l'ambiance et l'aspect de parure et de décoration des fontaines qui correspondent ainsi à la beauté des jeunes filles voir SABETAI 2009, p. 105.

37. SABETAI 2009, p. 105-106.

38. Pour une vue complète de la question voir MANFRINI ARAGNO 1992, déjà cité. On peut citer les exemples suivants d'hydrie: Hydrie, Florence, Museo Archeologico Etrusco 3792 ; CVA, Firenze, Regio Museo Archeologico 5, pl. 1881-1882. Voir MANAKIDOU 1992/3, p. 52, fig. 1. Hydrie du peintre AD, Wirzburg Universitat Martin Von Wagner Mus. 316 (L316). Cf. ABV, 334.2. Hydrie, Louvre 
Collection Campana 10651; CVA, Paris, Musée du Louvre 11, pl. 135, 1-4; LIMC VII, pl.400, Priamos 31 (S).

39. SABETAI 2009, p. 104, sur la question du topos iconographique fonctionnant comme une « métaphore de l'idéal féminin ».

40. Pour le détail des parures que portent les jeunes filles de l'hydrie du peintre AD, Boston MFA 61.195 ; voir notamment PEDLEY 1992, p. 144-145, et pour une vue d'ensemble voir p. 166, fig. 6.32.

41. Pour la présence de la fleur dans les mains des jeunes filles à la fontaine voir MANFRINI ARAGNO 1992, p. 134.

42. Parmi ces activités liées à la parure et la toilette, il y a aussi l'apprentissage de la beauté et de la féminité comme élément des qualités de l'épouse. On trouve aussi les gestes de consécration des jouets d'enfance des jeunes filles qui les remettent à Artémis ou Athéna. Voir MANFRINI ARAGNO 1992, p.135-136. Pour la question des danses qui ont lieu dans le cadre de la fontaine voir aussi MANAKIDOU 1992/3, p. 58.

43. Notamment sur les deux hydries du peintre AD, celle de Boston MFA 61.195, et aussi celle de Wirzburg Universitat Martin Von Wagner Museum L316.

44. Voir par exemple Hydrie, Berlin, Staatliche Museen 1843 ; cf. ABV 478, 85. Concernant la présence des sangliers renvoyant aux activités masculines, voir MANFRINI ARAGNO 1992, p. 131.

45. Cf. CVA, Londres British Museum 6, pl. 90.3, 93.1. Para., 165.74 ter ; Add. ${ }^{2}, 97$.

46. Lécythe, New York, Metropolitan Museum of Art, 56.11.1. cf. Para., 66; voir aussi MANFRINI ARAGNO 1992, p. 131-132.

47. La veille du mariage un jeune enfant apportait à la jeune femme l'eau du bain nuptial dans une hydrie, voir MANFRINI ARAGNO 1992, p. 137.

48. Une course de char est représentée sur la panse de l'hydrie du peintre AD, Boston MFA 61.195 ; sur l'hydrie de la Wurzburg Universitat, Martin von Wagner Museum L316; on peut supposer qu'il s'agit d'un départ de course de char. Le contexte de la scène sur l'épaule du vase du groupe de Léagros à Londres BM B335 est plus ambigu, la présence des chiens par exemple s'expliquerait mal dans le cadre d'un départ de course. Sur les différentes activités masculines représentées sur l'épaule de ces hydries, voir MANAKIDOU 1992/3, p. 67.

49. Parmi les diverses représentations de scènes de combat, ou liées à la guerre, on peut citer par exemple la représentation d'un combat de deux hoplites sur l'épaule d'une hydrie de Florence, Museo Archologico Etrusco 3792 ; ou encore un hoplite luttant contre deux cavaliers sur l'épaule d'une hydrie de Boston, MFA 01.8125, cf. CVA, Boston, Museum of Fine Arts 2, 16, pl. 74.1-3. Ou encore un départ de guerrier sur une autre hydrie du British Museum, Londres BM336; CVA Londres, British Museum 6, pl. 90.4, 93.2 ; cf. ABV, 266.3, 678 ; Add. ${ }^{2}$, 69. Voir le commentaire de M ANFRINI ARAGNO 1992, p. 145.

50. La violence ou les éléments érotiques de certaines représentations renvoyant probablement à une peur de la sexualité féminine. Sur ce point voir SABETAI 2009, p. 107-108.

51. Sur la question du rapt et de la violence exercée sur les jeunes filles à la fontaine, ainsi que sur l'utilisation de la représentation de Polyxène voir SABETAI 2009, p. 105 et 111. L'auteur considère aussi le cas d'Amymonè poursuivie par Poséidon, que je ne pourrai pas développer dans le cadre de cet article ; voir aussi MANFRINI ARAGNO 1992, p. 145.

52. Hydrie du groupe de Léagros, Londres BM B307 ; CVA, Londres, British Museum 6, pl. 76.4, 80.1 ; LIMC, Achilleus, 301, pl. 88 ; Cf. ABV, 361.17 ; et aussi Add. ${ }^{2}, 96$;

53. Hydrie, Londres, British Musuem, 99. 7-21.4, cf. ARV, 297, 15.

54. Pour les sources littéraires antiques qui ont abordé le thème de la mort de Troïlos voir notamment KNITTLMEAYER 1997, p. 80, qui discute la reprise du thème par les peintres sur les vases. Quant au silence d'Homère au sujet de cet épisode voir le commentaire de Pausanias, I, 22, 6. 
55. Cf. LIMC I, pl. 83, Achilleus 266 ; Para., 510 ; Add., 97 ; Add. ${ }^{2}, 195$; ARV ${ }^{2}, 210.174$. KNITTLMEAYER 1997, p. 81-82, pl. 19.1.

56. Skyphos de Brygos, Vienne Kunsthistorisches Museum 3710 ; CVA Vienne, Kunsthistorisches Museum 1, 29-31, pl. 35, 36, 37. Cf. Para., 366, 368. Voir aussi DUCREY 1985, p. 234, fig. 155 ; CARTER et MORRIS 1996, p. 456-457, pl. 28.10-12 ; KNITTLMEAYER 1997, pl. 6.1; LISSARRAGUE 2001, p. 98-99, pl. 77-78 ; LATACZ 2008, p. 384, nº126.

57. Homère, Iliade 24, 233.

58. Fond de coupe, successeur de Douris, cf. $A R V^{2}, 1670.58$ bis ; voir aussi KEPHALIDOU 1996, p. 207, pl. 45.Г 85 (dessin). De fait, les objets apportés par Priam à Achille ne sont pas seulement ceux du mariage comme le dit LISSARRAGUE 2001, p. 98, mais également ceux de la victoire dans un agôn; en tout état de cause ce sont les signes extérieurs de la valeur personnelle et familiale, du statut social de celui qui les possède.

59. Concernant le passage du statut d'éphèbe à celui de citoyen il faut tenir compte du fait qu'il y a une idéalisation de la jeunesse et de la beauté qui fait que les vainqueurs sont souvent représentés de manière juvénile.

\section{RÉSUMÉS}

L'article se propose d'étudier les différents contextes dans lesquels des hydries sont représentées afin d'étudier quelle pouvait être, au-delà de leur vocation pratique, leur valeur symbolique dans ces différents types de représentation. La présence des hydries dans les scènes de victoire aux concours, tout comme leur utilisation par des jeunes filles autour des fontaines, enfin leur emploi à des fins de tractation marchande, donnent à l'hydrie une pluralité d'emplois et d'usages qu'il convient de mettre en parallèle afin de déterminer chacun des sens que recouvre cet objet dans l'iconographie attique $d u v^{e}$ siècle av. n.è. et de chercher à comprendre dans quelle mesure l'hydrie, au-delà de sa polysémie, est également le signe d'un passage d'un état à un autre pour celui qui en a l'usage.

The article proposes to study the various contexts in which the hydrias are depicted beyond its obvious practical use. For example, the hydria is used in different contexts such as the agonistic representations of victory, the nymphai at the fountain or as a valuable object used to pay a ransom. In each case, the hydrias have a plurality and obvious use, which need to be treated in parallel in order to determine each and every sense which covers this object in the attic iconography of the $5^{\text {th }}$ B.C. On top of this, it is important to understand in what way this vase has an obvious polysemical use, and is also a sign of passage from one state to another for the person using it.

\section{INDEX}

Mots-clés : hydrie, concours, prix, récompense, athlète, femme, transition, vase

Keywords : hydria, contest, prize, reward, athlet, woman, passage 
AUTEUR

GWENOLA COGAN

ANHIMA - UMR 8210 